\title{
Osteosarcoma to the left Heart
}

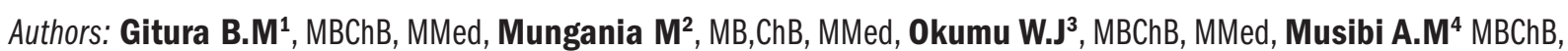
MMed, Gatura J.G5 ${ }^{5}$ MBChB Affiliation 1. Department of Medicine, Kenyatta National Hospital (KNH), 2. Department of pathology, KNH 3. Department of cardiothoracic surgery, KNH, 4. Kenya Medical Research Institute. 5. Department of Surgery, University of Nairobi, Correspondence Dr Gitura BM, Department of Medicine,KNH, P.0 Box 2249-00202 Nairobi Kenya.

\section{Summary}

Primary and secondary tumors of the heart are rare. Myxoma is the commonest benign tumor. Of the malignant lesions metastatic heart tumors are more common although these have received little clinical attention. The right side of the heart is more commonly involved while the pericardium and/or myocardium more frequently involved than the endocardium. We present a case of metastatic osteosarcoma into the endocardium of the left atrium.

\section{Introduction}

Primary tumors of the heart are rare across all age groups; with a reported prevalence of 0.001 to 0.03 percent in autopsy series (1). The potentially lethal course and the possibility of cure with adequate excision make early diagnosis and optimal therapeutic attainment crucial. Tumors that metastasize to the heart from other organs are 100-1000 fold more common than primary tumors (2) with an incidence of $1.23 \%$ in autopsy reports (3). Intracavitary growth of secondary heart tumors, however is unusual. In children, metastatic cardiac tumors are associated with Wilms' tumors, neuroblastoma, and nonHodgkin's lymphoma (4). Only rarely have osteogenic sarcoma metastasis to the heart been reported (5).

\section{Case Report}

A 17 year old African male was admitted to Kenyatta National Hospital with two months history of worsening dyspnoea, cough and four days of left sided weakness. He had lost significant weight, but had no fevers or night sweats. He had no prior rheumatic heart disease and no history of calf pain. His past medical history was significant for a right above knee amputation for osteogenic sarcoma six months prior to his current presentation. He had not been started on chemotherapy at the time. Examination revealed a young man in fair general condition, mild pallor, afebrile with mild leg oedema and no lymphadenopathy. His vital signs were normal. He had a healed right above knee stump. Neurological examination revealed normal higher functions, seventh cranial nerve palsy (upper motor neuron type), and a left sided weakness (power grade $0 \mathrm{MRC}$ scale) in both upper and lower limbs. The right upper limb had normal power. His cardiovascular examination revealed a regular radial pulse, raised jugular venous pressure, non displaced apex with an apical systolic murmur grade $4 / 6$ and basal crackles. He had a tender hepatomegaly.

Investigations revealed a normal hemogram, urea, creatinine and electrolytes. Chest X-ray showed increased vascular markings in the pulmonary vasculature but no obvious masses. There was fullness of the pulmonary bay but the cardiothoracic ratio was normal. No double shadows were noted (figure 1)

Electrocardiogram revealed a sinus rhythm with tachycardia, transthoracic echocardiogram showed a large left atrial mass that prolapsed into the left ventricle in diastole (figure 2). The left ventricular chamber size was normal with ejection fraction of 53\%. A CT scan of the brain showed an extensive right parietal infarct in the region of middle cerebral artery. No mass lesion was seen.

Initial diagnosis of left atrial thrombus with possible embolic ischemic stroke was made and treatment started with intravenous unfractionated heparin with a target activated partial thromboplastin time of 50- 70s. A discussion of the echocardiogram findings with the cardiothoracic team agreed on an emergency open heart surgery to evacuate the thrombus.

The surgery revealed a soft grey tumor in the left atrium arising from the intima of the left superior pulmonary vein and extending into the left ventricle through the 


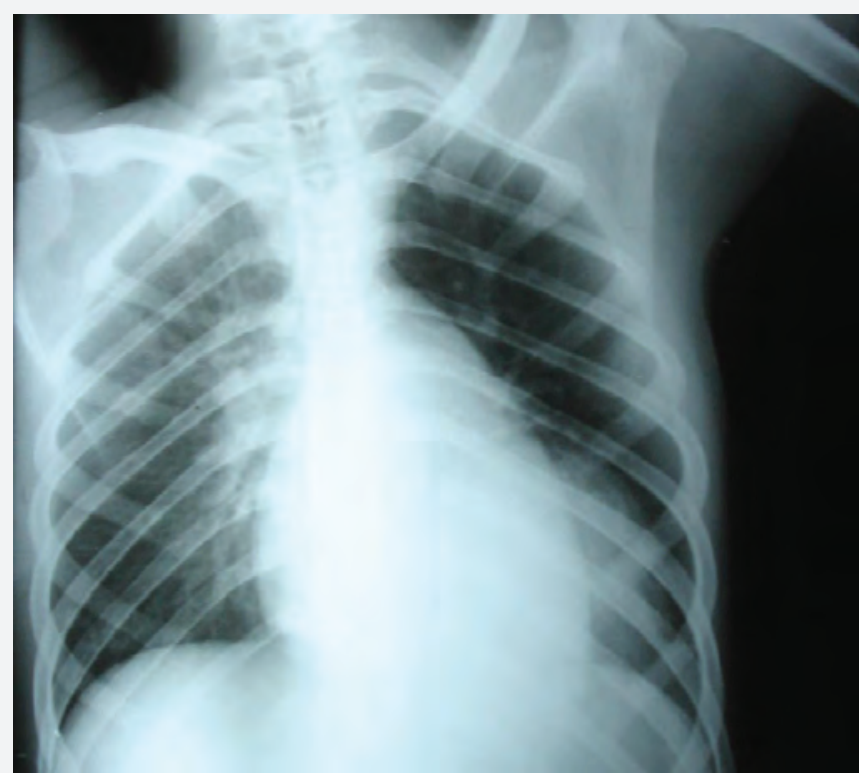

Figure 1 chest $x$-ray-note the increased vascular markings and fullness of pulmonary bay. No calcifications demonstrated.

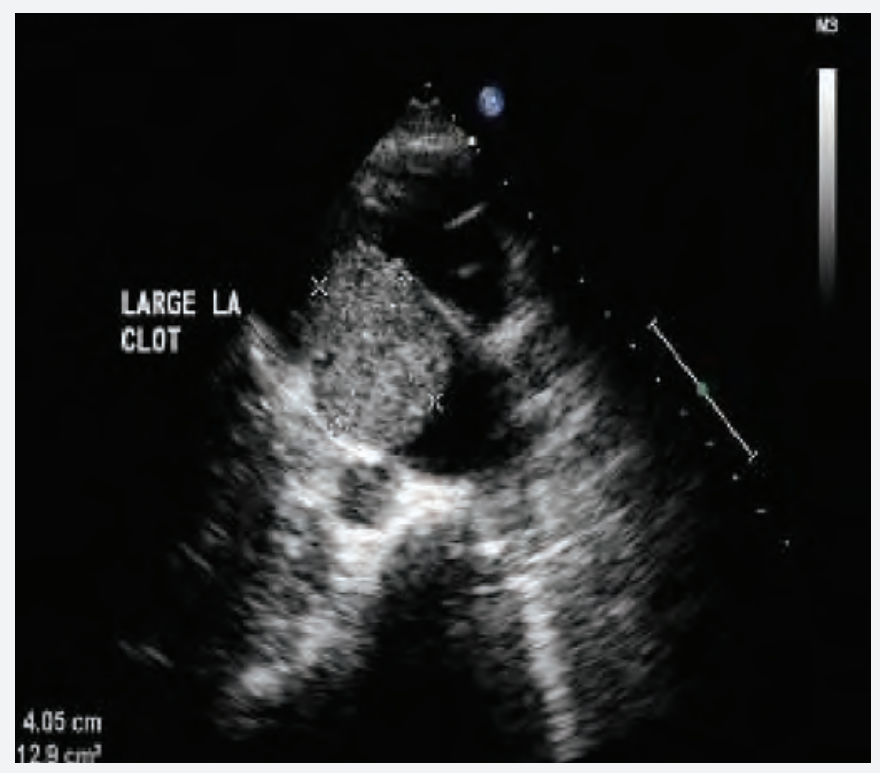

Figure 2 Echocardiogram showing a large left atrial mass of mixed echogenicity mitral orifice (Fig. 3) near complete excision was performed.

The excised specimen was whitish polypoid soft tissue, gelatinous in nature, with necrotic, haemorrhagic and cystic areas (Figure 4). On microscopy, it was a nodular tumor composed of lobules of malignant cartilage with peripheral spindling. It had fine ramifying osteoid matrix between tumor cells, features in keeping with a chondroblastic osteosarcoma (Figure 5 and 6).

The patient was started on adjuvant chemotherapy that included cisplatin, adriamycin, vincristine, and endoxan.

\section{Discussion}

The heart may be involved by primary as well metastatic tumors. Primary tumors of the heart are very rare $(<0.03$ $\%$ in postmortem series) (1) with myxoma being most frequent. Metastases from neoplasms arising in other organs are far more common and include carcinomas, sarcomas, hematologic malignancies and melanomas. The most common means of spread is via the blood stream through coronary arteries and sub-diaphragmatic venacava (6 ).They can also reach the heart through lymphatic channels, direct extension from adjacent lung, breast, esophageal and thymic tumors (7). Nonetheless cardiac metastasis are infrequent. Possible explanations for relative protection of the heart from metastases include the continuous forceful stoke of the heart, rapid blood flow, the afferent lymphatic circulation or a combination of all these and other unknown factors.

Metastatic spread to the heart has been identified in approximately one-fifth of all patients who have metastatic cancer, with lung carcinoma being most common primary tumor (2). Melanoma is commonly referred to as the neoplasm with the greatest propensity for cardiac involvement, with about $50 \%$ of patients found after death to have had cardiac involvement (8). Most cardiac metastasis involve the right side and only rarely is the left heart involved $(3,4)$. Osteosarcomas are characterized by the ability of both the primary tumor and distant metastasis to produce osteid tissue. Rees et al found that pulmonary metastases were clearly visible on radiology (9). However a chest radiograph on our patient was negative for calcification. The prognosis for metastatic osteosarcoma is generally poor but may be improved by early diagnosis and the addition of chemotherapy, radiotherapy and surgical resection (10). Trans thoracic and trans 


\section{Unusual Metastasis to the Heart}

Gitura B.M, Mungania M, Okumu W.J, Musibi A.M, Gatura J.G

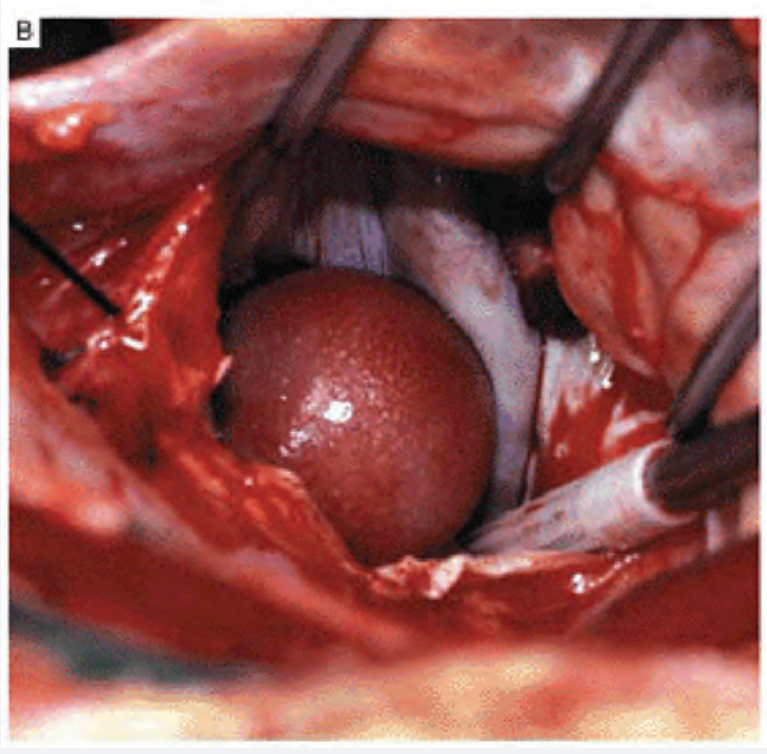

Figure 3 Mass in the left atrium

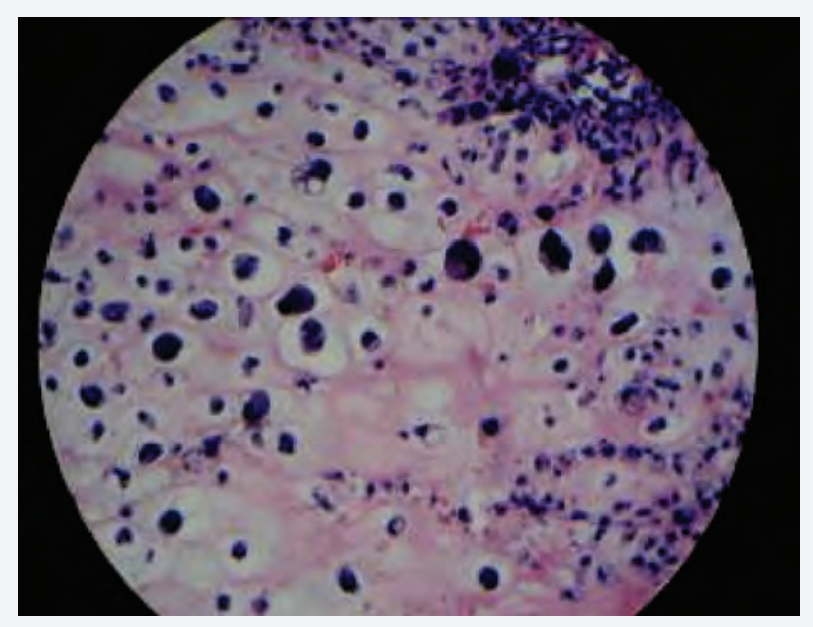

Figure 5 Chondroblastic cells and fine ramifying osteoid matrix

oesophageal echocardiography remain the procedure of choice in screening and fully elucidating the position and size of cardiac tumors (11). It also provides real-time portrayal of those extremely mobile tumors $(11,12)$. Their easy prolapse through the atrio-ventricular valve may cause hemodynamic compromise, illustrating the need for surgery before attaining large size and causing

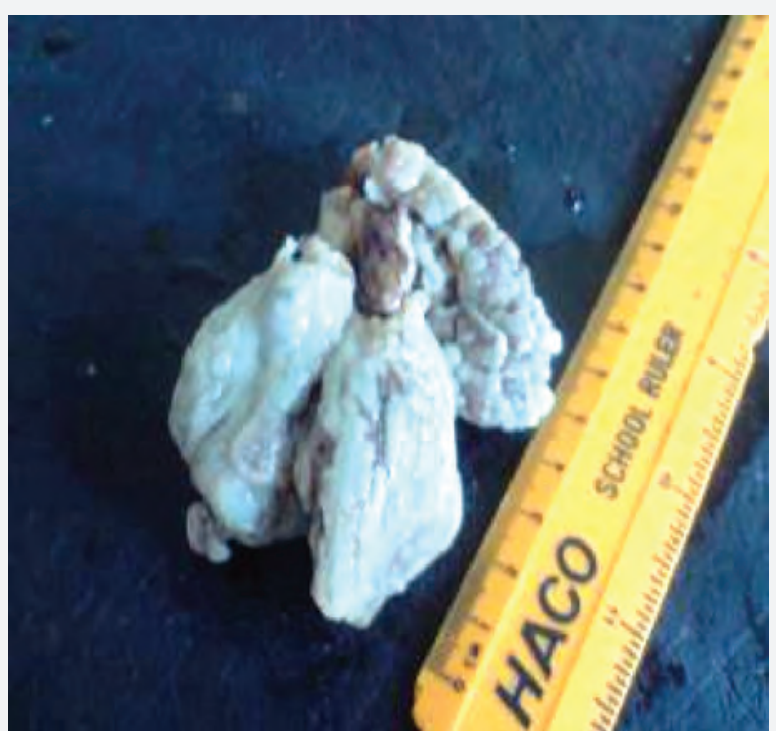

Figure 4 Excised mass after formalin preservation

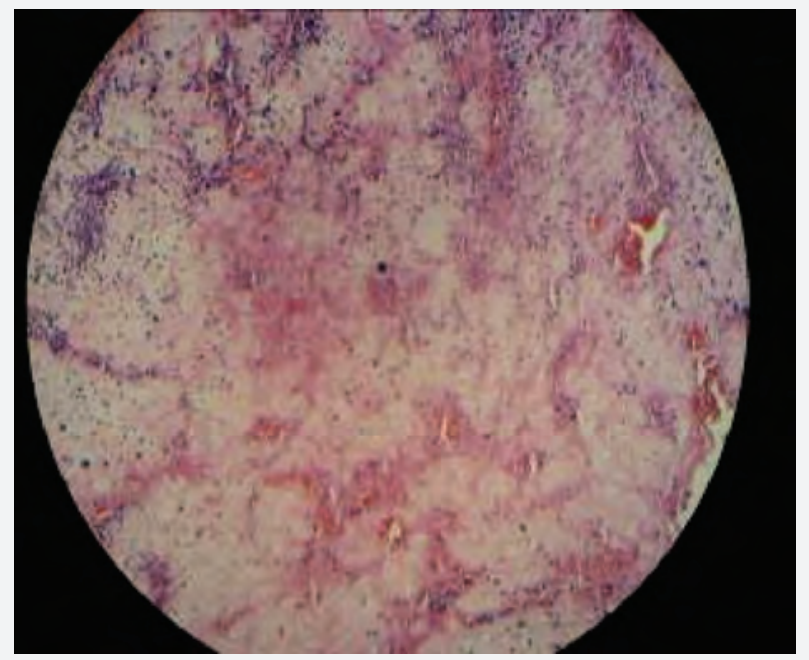

Figure 6 Necrotic and hemorrhagic cystic areas

obstruction or embolisation. Our patient presented with features of heart failure and embolic brain infarct with what was initially thought to be a left atrial thrombus. Although surgery is contraindicated in the presence of metastatic disease, the role of surgical palliation of intracaval and intracardiac tumor to relieve obstruction and prevent embolization is justified $(11,13)$. Our patient 
underwent emergency open heart surgery with near total resection of the tumor. His hemodynamic parameters improved rapidly but his paralysis persisted. At the time of writing this article the patient has remained hemodynamically stable with persistent left hemiparesis despite aggressive physiotherapy for six months.

In conclusion, cardiac neoplasms whether primary or secondary, benign or malignant,myxomas or sarcomas are clinically significant lesions with life threatening sequelae that affect patients of all ages. Most benign tumors can be resected completely with excellent prognosis. Many malignant tumors cannot be resected completely. Palliation, radiation and chemotherapy may be used but without great expectations.

\section{References}

1. Burke A, Virmani R: Fascicle 16,3rd series:Tumors of the heart and great vessels, 1996

2. Sarjeant JM, Butany J,Cusimano RJ: Cancer of the heart:Epidemiology and management of primary tumors and metastasis.Am J cardiovasc drugs.2003; 3:407-421.

3. Lam KY,Dickens P,Chan AC.Tumors of the heart.A 20 year experience with a review of 12485 consecutive autopsy cases.Arch Pathol Lab Med 1993;117:1027-1031

4. Vander TJ. Unusual tumors of the heart. Semin Thorac Car- diovasc Surg $2000 ; 12: 89-100$

5. Shaffique QS, Rajesh CM, Rakesh SN. Cardiac metastasis of osteosarcoma. J Coll Physicians Surg Pak 2004;14:430 432.

6. Tsuji Y, Yamashita C, Wakiyama H et al. Surgical treatment for transvenous tumor extension into the heart: four cases. J Vasc Surg (1998) 27(4): 740 - 744.

7. Daneman A, Martin DJ, Chan HSL. Cardiac metastasis from osteosarcoma. A report of two cases. JCAT (1983);7:41 - 43.

8. Petropoulakis PN,Steriotis JD,Melanidis JG, Metastatic malignant melanoma as an intracavitary mass in the right heart.Eur J Cardioth. Surg 1998;14:538-540.

9. Rees CR, Siddiqui AR, Ducret R. The role of bone scintigraphy in osteogenic sarcoma. Skeletal Radiol, 1986;15:365 $-367$.

10. Burke AP, Cowan D, Virmani R. Primary sarcomas of the heart. Cancer 1992;69:387 - 395

11. Salcedo EE, Cohen G, White RD et al. Cardiac tumors: Diagnosis and management. Curr Probl Cardial, 1992; 17: 73-173

12. Selbert KA, Rettenmier CW, Waller BF, et al. Osteogenic sarcoma metastasis to the heart. Am J Med. 1982; 73: 136-141

13. Magovern GJ, Yusuf MF, Liebler GA, et al. The surgical resection and chemotherapy of osteogenic sarcoma of the right ventricle. Ann Thorac Surg 1989;29:76 - 80 . 